Nouvelles perspectives en sciences sociales

Revue internationale de systémique complexe et d'études relationnelles

\title{
Validation d'un schème national sur le développement des services de santé pour les communautés francophones et acadiennes en situation minoritaire au Canada : arrimage à des assises théoriques et modélisation
}

\section{Louis Giguère}

Volume 9, numéro 2, mai 2014

URI : https://id.erudit.org/iderudit/1025976ar

DOI : https://doi.org/10.7202/1025976ar

Aller au sommaire du numéro

Éditeur(s)

Prise de parole

ISSN

1712-8307 (imprimé)

1918-7475 (numérique)

Découvrir la revue

Citer cet article

Giguère, L. (2014). Validation d'un schème national sur le développement des services de santé pour les communautés francophones et acadiennes en situation minoritaire au Canada : arrimage à des assises théoriques et modélisation. Nouvelles perspectives en sciences sociales, 9(2), 207-236. https://doi.org/10.7202/1025976ar
Résumé de l'article

Nous avons présenté (Giguère, 2013), pour les communautés francophones hors Québec (CFASM), un schème juridictionnel national qui illustre l'offre et la demande de services de santé en français en fonction d'une masse critique de francophones qui reflète la vitalité linguistique de ces communautés. Nous corroborons ce schème à l'aide de procédures statistiques dont l'une identifie une forte association entre le rapport offre/demande (un ratio) et des facteurs qui reflètent la cohérence linguistique et culturelle des deux communautés de langue officielle. Ce résultat est cohérent avec le cadre de vitalité linguistique déjà proposé (Giguère, 2013), et il fait écho au travail de terrain de Forgues, Bahi et Michaud (2011) et aux modèles psycho-langagiers et d'autonomie culturelle de Deveau, Landry et Allard (2009), de Landry, Allard et Deveau $(2007,2008)$ et de Landry (2012). Nous modélisons le développement des services de santé en français pour les CFASM dans un cadre national structurant qui est cohérent avec ces assises empiriques et théoriques. Cette modélisation suggère deux approches stratégiques distinctes mais complémentaires pour favoriser le développement des services de santé en français. 


\section{Validation d'un schème national sur le développement des services de santé pour les communautés francophones et acadiennes en situation minoritaire au Canada : arrimage à des assises théoriques et modélisation}

Louis Giguère

RésoSanté Colombie-Britannique, Vancouver

\section{Introduction}

D ans une publication précédente ${ }^{2}$, nous quantifions et nous bonifions le schéma du CCCFSM ${ }^{3}$ sur le développement des services de santé pour les communautés francophones et acadiennes en situation minoritaire du Canada (CFASM) en

1 Errata: Dans l'article qui précède celui-ci, soit dans le volume 9, numéro 1, p. 319-345, de NPSS : 1) à la page 330, au lieu de "Équation 1 : Demande $=23,55+71,78 *$ facteur 6 ", on lira "Équation $1:$ Demande $=23,55+$ $71,78 *$ facteur $5 » ; 2$ ) à la page 330, au lieu de «Équation $2:$ Offre $=-5,40$ $+98,87 *$ facteur 6 ", on lira "Équation $2:$ Offre $=-5,40+98,87 *$ facteur 5 »; 3) à la page 334, $2^{\text {e }}$ paragraphe, lignes 6 et 7 , au lieu de " (le NouveauBrunswick avec 0,91), on lira " (le Nouveau-Brunswick avec 0,87)».

2 Louis Giguère, "Les services de santé en français pour les communautés francophones et acadiennes en situation minoritaire du Canada : bonification du schéma du CCCFSM (2001) ", Nouvelles perspectives en sciences sociales, vol. 9, $\mathrm{n}^{\circ}$ 1, 2013, p. 319-345.

3 CCCFSM [Comité consultatif des communautés francophones en situation minoritaire], "Pour un meilleur accès à des services de santé en français ", Ottawa, Fédération des communautés francophones et acadiennes du Canada, 2001. 
nous basant sur des données probantes. Pour ce faire, nous portions en graphique sur une base juridictionnelle territoriale et provinciale des indicateurs d'offre et de demande de services en santé en français provenant de l'Enquête sur la vitalité des minorités de langues officielles (EVMLO) de Statistique Canada ${ }^{4}$ sur l'axe des « $\mathrm{Y}$ » et un indicateur de masse critique de francophones provenant du recensement national de $2006^{5}$ sur l'axe des « $\mathrm{X}$ ». En dépit des variations socio-démographiques considérables qui existent d'une juridiction à l'autre ${ }^{6}$, l'offre et la demande de

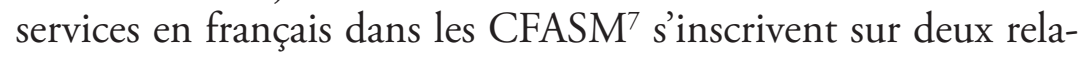
tions linéaires qui illustrent un schème national cohérent.

$4 \quad$ Sylvie Lafrenière, « L'accès aux services de soins de santé : le potentiel analytique de l'Enquête sur la vitalité des minorités de langue officielle ", dans Le Secrétariat national du Consortium national de formation en santé Société, langue et santé : les barrières à l'accès aux services de santé pour les communautés francophones minoritaires, $2^{\mathrm{e}}$ Forum national de recherche sur la santé des communautés francophones en situation minoritaire, Ottawa, 2008, p. 31-32.

5 Canada, Statistique Canada, « Portrait des communautés de langue officielle au Canada : recensement de 2006 ", Ottawa, 2009, DVD.

6 CNFS et SSF [Consortium national de formation en santé et Société Santé en français], «Introduction et mise en contexte », dans Le Secrétariat national du Consortium national de formation en santé Société, langue et santé : les barrières à l'accès aux services de santé pour les communautés francophones minoritaires, 2e Forum national de recherche sur la santé des communautés francophones en situation minoritaire, Ottawa, 2008, p. 15-18.

7 Nous faisons référence aux CFASM sur une base juridictionnelle, soit la CFASM du Nouveau-Brunswick, la CFASM de l'Alberta, la CFASM du Nunavut, etc. 


\section{Figure 1}

Le schème du développement des services de santé pour les communautés francophones en situation minoritaire du Canada (schéma CCCFSM bonifié).

Offre = « carrés $»$; demande $=$ « cercles $»$

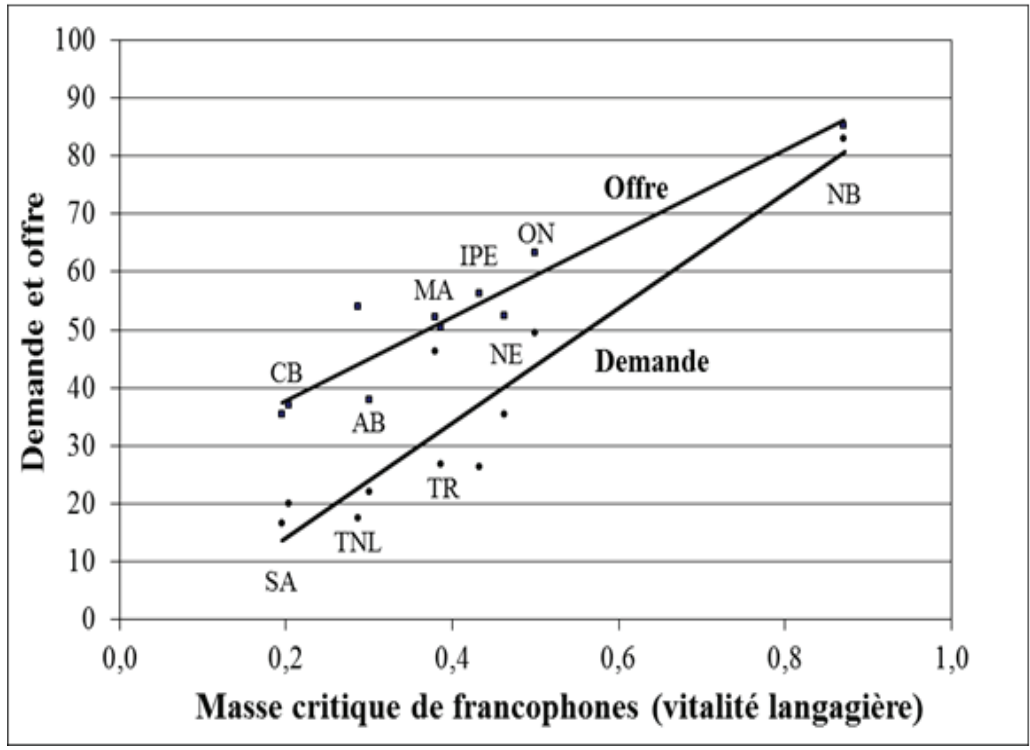

Ce schème tient compte de $87 \%$ et $90 \%$ de la variation de l'offre et de la demande de services de santé en français respectivement et s'inscrit dans le contexte d'un indicateur de masse critique qui reflète la vitalité langagière des CFASM. L'offre et la demande seraient plus grandes lorsque la vitalité langagière des CFASM est grande, mais de telle façon que l'offre est systématiquement moindre que la demande, et ce, d'autant plus que la vitalité langagière de ces communautés est faible (la pente de la relation linéaire pour l'offre est alors plus à pic). Le schème national présenté dans la figure 1 nous offre donc une représentation concrète du développement de l'offre et la demande de services en français dans les CFASM mais cette représentation repose sur une transformation quantitative de données sur la perception des francophones. Pour corroborer ces résultats, nous 
cherchons dans cet article à (a) établir une plus grande validité statistique pour les différences d'offre et de demande observées, et (b) développer un contexte d'interprétation plus large en identifiant quels facteurs socio-démographiques sont associés à ces différences dans le but de développer un contexte d'interprétation confirmateur et d'arrimer nos résultats empiriques à des assises théoriques. Cet arrimage établira une consistance entre le schème observé et le travail de terrain de Forgues, Bahi et Michaud $^{8}$ et les modèles de développement psycholangagier et d'autonomie culturelle de Deveau, Landry et Allard', de Landry, Allard et Deveau ${ }^{10}$ et de Landry ${ }^{11}$. Nous effectuerons ensuite une modélisation qui repose sur un cadre national structurant qui est cohérent avec ces assises empiriques et théoriques et qui éclaire la pratique des réseaux de santé en français des CFASM.

\section{Méthodes}

Nous nous demandons au préalable si les observations (a) « que l'offre de services en français dans les CFASM est moindre que la demande » est statistiquement significative, et (b) « qu'il existe un plus grand écart offre/demande lorsque la vitalité langagière est faible » sont statistiquement significatives. La méthode utilisée consiste à établir, à l'aide du test d'hypothèse statistique $t$, s'il existe une différence de pentes entre les relations de l'offre et de

8 Éric Forgues, Boniface Bahi et Jacques Michaud, "L'offre de services en français en contexte francophone minoritaire ", Ottawa, Institut canadien de recherche sur les minorités linguistiques, 2011.

9 Kenneth Deveau, Rodrigue Landry et Réal Allard, "Utilisation des services gouvernementaux de langue française. Une étude auprès des Acadiens et francophones de la Nouvelle-Écosse sur les facteurs associés à l'utilisation des services gouvernementaux en français ", Ottawa, Institut canadien de recherche sur les minorités linguistiques, 2009.

10 Rodrigue Landry, Réal Allard et Kenneth Deveau, «A Macroscopic Intergroup Approach to the Study of Ethnolinguistic Development ", International Language of the Sociology of Language, vol. 185, 2007, p. 225-253; Rodrigue Landry, Réal Allard et Kenneth Deveau, "Un modèle macroscopique du développement psycholangagier en contexte intergroupe minoritaire ", Diversité urbaine, hors série, automne 2008, p. 45-68.

11 Rodrigue Landry, "Autonomie culturelle, cultures sociétales et vitalité des communautés de langue officielle en situation minoritaire au Canada ", Minorités linguistiques et société, $\mathrm{n}^{\circ}$ 1, 2012, p. 159-179. 
la demande ou s'il existe une différence d'élévation le long de l'axe des "Y $»^{12}$. Ayant établi qu'il existe une différence significative, nous identifions ensuite, à l'aide d'une procédure statistique de régression linéaire par étape ${ }^{13}$, quels facteurs socio-démographiques (tableau $1^{14}$ ) sont associés avec l'écart relatifi' ${ }^{15}$ qui existe entre l'offre et la demande, dans le but de développer un contexte d'interprétation élargi. Selon cette méthode de régression, la procédure choisit un premier facteur, parmi ceux qui se trouvent au tableau 1, en raison de sa plus grande association statistique avec le rapport offre/demande; puis elle en choisit un suivant tout en tenant compte du premier facteur, de sorte que la procédure identifie, dans l'ordre, l'ensemble des facteurs statistiquement importants qui tiennent compte de la variation du rapport offre/ demande. Le seuil de signification statistique choisi est celui utilisé communément, soit $5 \%$ (ou $\alpha=0,05$ ).

$\overline{12}$ Jerrold H. Zar, Biostatistical Analysis, $2^{\mathrm{e}}$ édition, Upper Saddle River (NJ), Prentice Hall, 1984, p. 296-297.

13 Jerrold H. Zar, ibid., p. 341-344.

14 Les territoires sont exclus pour cette procédure parce que certaines données ne sont pas disponibles territoire par territoire.

15 Relatif en ce sens que cet écart est exprimé sous forme d'un rapport de l'offre sur la demande (un ratio). 


\section{Tableau 1}

Facteurs socio-démographiques (variables indépendantes) utilisés pour la procédure statistique de régression linéaire par étape du rapport offre/demande (pour 9 provinces canadiennest, sauf le Québec).

\begin{tabular}{|c|c|}
\hline $1-$ & Nombre de francophones, dans une juridiction \\
\hline $2-$ & $\begin{array}{l}\text { Densité de francophones, dans une juridiction (1- ci-dessus divisé par la superficie } \\
\text { du territoire en } \mathrm{km}^{2} \text { ) }\end{array}$ \\
\hline 3- & $\begin{array}{l}\text { Proportion de francophones, dans une juridiction (1- ci-dessus divisé par la } \\
\text { population totale de la juridiction) }\end{array}$ \\
\hline 4- & $\begin{array}{l}\text { Nombre de francophones qui parlent le plus souvent français à la maison, dans une } \\
\text { juridiction }\end{array}$ \\
\hline 5- & $\begin{array}{l}\text { Proportion de francophones qui parlent le plus souvent français à la maison, dans } \\
\text { une juridiction (4-ci-dessus divisé par } 1 \text { - ci-dessus) }\end{array}$ \\
\hline 6- & Nombre de langues parlées, dans une juridiction \\
\hline 7- & $\begin{array}{l}\text { Indice de diversité de Simpson pour les langues parlées, dans une juridiction ; une } \\
\text { mesure globale de la diversité linguistique } ; \sum\left(n_{i}\left(n_{i}-1\right) / N(N-1) \text { où « } n_{i} » \text { représente }\right. \\
\text { le nombre d'individus parlant le langage « } i » \text { et «N } N \text { représente le nombre total } \\
\text { d'individus }\end{array}$ \\
\hline 8- & $\begin{array}{l}\text { Proportion d'immigrants francophones par rapport à la population francophone, dans } \\
\text { une juridiction* } \dagger\end{array}$ \\
\hline 9- & Proportion de la population bilingue, dans une juridiction (deux langues officielles) \\
\hline $10-$ & Proportion d'anglophones, dans une juridiction \\
\hline 11- & Âge médian de la population, dans une juridiction \\
\hline 12- & Taux d'emploi, dans une juridiction (\%) \\
\hline 13- & $\begin{array}{l}\text { Proportion d'individus qui ont reçu une formation postsecondaire, dans une } \\
\text { juridiction }\end{array}$ \\
\hline 14- & Proportion d'individus qui représentent une minorité visible, dans une juridiction \\
\hline 15- & Revenu moyen, dans une juridiction \\
\hline $16-$ & Proportion d'individus qui travaillent à plein temps, dans une juridiction \\
\hline
\end{tabular}

* René Houle et Jean-Pierre Corbeil, « Portrait statistique de la population immigrante de langue française à l'extérieur du Québec (1991 à 2006) », Ottawa, Statistique Canada, 2010.

† Certaines données ne sont pas disponibles territoire par territoire.

Source, sauf indication autre : Canada, Statistique Canada, "Portrait des communautés de langue officielle au Canada : recensement de 2006 ", Ottawa, 2009, DVD. 


\section{Résultats}

Première étape : Validation statistique de l'observation que l'offre de services en français dans les CFASM est moindre que la demande

Nous vérifions d'abord si dans la figure 1 la pente pour l'offre est plus grande que la pente pour la demande. L'hypothèse de travail est $\mathrm{H}_{1}: \mathrm{m}_{\text {offre }}>\mathrm{m}_{\text {demande }}$, et l'hypothèse nulle $\mathrm{H}_{0}: \mathrm{m}_{\text {offre }} \leq \mathrm{m}_{\text {demande }}$ ( $\mathrm{m}$ " représentant la pente d'une droite de régression). Le résultat n'est pas statistiquement significatif $(\mathrm{t}=-1,612, \mathrm{ddl}=$ $16, p=0,063)$, mais il est très près du seuil de signification choisi $(\alpha=0,05$; à comparer avec $p=0,063)$. Comme accepter $\mathrm{H}_{0}$ dans un test d'hypothèse où $\mathrm{p}=0,063$ comporte un risque élevé d'une erreur de type II (c'est-à-dire accepter $\mathrm{H}_{0}$ lorsque $\mathrm{H}_{0}$ est fausse), nous concluons qu'il existe un écart grandissant entre l'offre et la demande avec la faiblesse de la vitalité linguistique, bien que cet écart ne soit pas statistiquement significatif au seuil de signification choisi $(\alpha=0,05)$.

Nous vérifions ensuite si le niveau d'offre est moindre que celui de la demande, le long de l'axe des « $Y$ » (l'hypothèse de travail est $\mathrm{H}_{1}$ : demande $>$ offre et l'hypothèse nulle est $\mathrm{H}_{0}$ : demande $\leq$ offre). Le résultat est statistiquement significatif au seuil choisi $(t=-6,042, d d l=17, p<0,001)$. Nous concluons donc que l'offre de services de santé en français est significativement moindre que la demande.

Deuxième étape : validation des variables socio-démographiques qui sont le plus fortement associées avec le rapport offre/demande

Comme la méthode de régression devait considérer dès le départ deux facteurs qui sont très fortement associés l'un avec l'autre, soit la proportion de la population qui est anglophone et l'indice de diversité de Simpson ${ }^{16}$ sur la première langue parlée à la maison, nous présentons séparément deux procédures de régression aux tableaux 2 et 3; la première procédure inclut la propor-

Edward H. Simpson, « Measurement of Diversity », Nature, n 163, 1949, p. 688. 
tion de la population qui est anglophone à l'exclusion de l'indice de diversité de Simpson sur la première langue parlée à la maison, et vice versa pour la deuxième procédure.

\section{Tableau 2}

Régression linéaire des facteurs démographiques du tableau 1 sur le rapport offre/demande de services de santé en français (procédure partant de la proportion de la population qui est anglophone)*

(Pour l'équation dans son ensemble, $\mathrm{F}_{2 ; 6}=14,89 ; \mathrm{p}=0,005$ )

\begin{tabular}{|l|c|c|c|}
\hline & Coefficients & $R^{2}$ & $p$ \\
\hline Sur l'axe des Y & 1,62 & & 0,002 \\
\hline $\begin{array}{l}\text { Proportion de la population qui est } \\
\text { anglophone, dans une juridiction }\end{array}$ & $-0,868$ & 0,568 & 0,002 \\
\hline $\begin{array}{l}\text { Proportion de la population } \\
\text { francophone qui est immigrante, dans } \\
\text { une juridiction }\end{array}$ & $-0,008$ & 0,832 & 0,022 \\
\hline
\end{tabular}

* = l'indice de diversité de Simpson sur la langue parlée le plus souvent à la maison est exclu de cette procédure

\section{Tableau 3}

Régression linéaire des facteurs démographiques du tableau 1 sur le rapport offre/demande de services de santé en français (procédure partant de l'indice de diversité de Simpson sur la première langue parlée à la maison)*

(Pour l'équation dans son ensemble, $\mathrm{F}_{2 ; 6}=20,519 ; \mathrm{p}=0,002$ )

\begin{tabular}{|l|c|c|c|}
\hline & Coefficient & $\mathrm{R}^{2}$ & $\mathrm{p}$ \\
\hline Sur l'axe des Y & 0,739 & & 0,001 \\
\hline $\begin{array}{l}\text { Indice de diversité de Simpson sur la } \\
\text { langue parlée le plus souvent à la } \\
\text { maison, dans une juridiction }\end{array}$ & $+0,608$ & 0,540 & \\
\hline $\begin{array}{l}\text { Proportion de la population francophone } \\
\text { qui est immigrante, dans une juridiction }\end{array}$ & $-0,926$ & 0,872 & 0,007 \\
\hline
\end{tabular}

${ }^{*}=$ la proportion de la population qui est anglophone est exclue de cette procédure 
Les deux procédures de régression proposent soit la proportion de la population qui est anglophone (première procédure; effet négatif), soit l'indice de diversité de Simpson (deuxième procédure; effet positif) comme facteur expliquant la plus grande proportion de la variation $\left(R^{2}=0,568\right.$ ou 0,540 respectivement $)$. Ce résultat est cohérent avec le fait que les deux provinces qui ont le plus petit rapport offre/demande (Terre-Neuve et Labrador, Île-du-Prince-Édouard) ont les plus grands pourcentages de population anglophone (tableau 4) alors que les deux provinces qui ont le plus grand rapport offre/demande (l'Ontario et le Nouveau-Brunswick) ont les plus petits pourcentages de population anglophone (en raison d'une forte représentation d'immigrants dans le cas de l'Ontario et d'une forte représentation de francophones dans le cas du Nouveau-Brunswick) ${ }^{17}$. Là où les anglophones sont dominants (et par conséquent où la diversité linguistique est moins grande par rapport à la communauté de langues officielles majoritaire), le rapport offre/demande est plus petit.

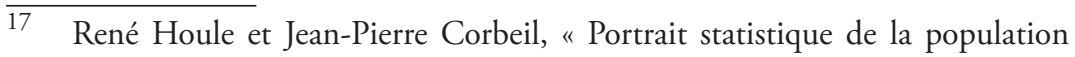
immigrante de langue française à l'extérieur du Québec (1991 à 2006) ", Ottawa, Statistique Canada, 2010. 


\section{Tableau 4}

Proportion de la population anglophone dans une juridiction et proportion de la population francophone qui est immigrante dans une juridiction

\begin{tabular}{|c|c|c|c|}
\hline $\begin{array}{l}\text { Proportion de population anglophone, } \\
\text { dans une juridiction }\end{array}$ & $\begin{array}{l}\text { Proportion de la population francophone } \\
\text { qui est immigrante, dans une juridiction }\end{array}$ \\
\hline Juridiction & & Juridiction & \\
\hline NNL & 0,979 & CB & 0,240 \\
\hline IPE & 0,964 & ON & 0,128 \\
\hline NE & 0,947 & AB & 0,127 \\
\hline SA & 0,902 & TNL & 0,105 \\
\hline CB & 0,825 & MA & 0,051 \\
\hline MA & 0,808 & SA & 0,047 \\
\hline$A B$ & 0,721 & NE & 0,041 \\
\hline ON & 0,712 & PEE & 0,020 \\
\hline NB & 0,684 & NB & 0,015 \\
\hline
\end{tabular}

TNL : Terre-Neuve et Labrador ; NE : Nouvelle-Écosse ; IPE : Île-du-Prince-Edward ; NB : Nouveau-Brunswick ; ON : Ontario ; MA ; Manitoba ; SA ; Saskatchewan ; AB ; Alberta ; CB :

Colombie-Britannique.

Les deux procédures de régression proposent ensuite un deuxième facteur expliquant une proportion significative de la variance, le même facteur dans les deux cas, soit la proportion de la population francophone qui est immigrante (tableaux 2 et $3 ; R^{2}=0,264$ et 0,332 pour la première et la deuxième procédures respectivement). Le fait que ce facteur a un effet négatif sur le rapport offre /demande ${ }^{18}$ pourrait surprendre mais

18 En Colombie-Britannique, la juridiction où plus de $25 \%$ de la communauté francophone est immigrante, les représentants de quatre communautés immigrantes qui participaient à un dialogue sur l'immigration francophone organisé par la Fédération des francophones de la C.-B. le 7 juin dernier ont exprimé un manque d'intérêt par rapport à la francophonie canadienne et au dossier des langues officielles. Les participants ont exprimé un plus grand attachement à la communauté anglophone majoritaire qui leur offre plusieurs services et à laquelle ils tentent de s'intégrer. Ils ont tous exprimé un attachement singulier à leur propre " code culturel » de sorte que la cohérence linguistique et culturelle de la communauté francophone demeure un défi étant donné que la francophonie internationale est très diversifiée. 
ce résultat est cohérent dans un contexte franco- et britannocolombien. Au total, les deux premiers facteurs de régression tiennent compte d'une proportion considérable de la variance totale $\left(\mathrm{R}^{2}:=0,832\right.$ et 0,872 respectivement), ce qui représente une forte association.

Les facteurs qui sont fortement associés avec le ratio offre/ demande sont donc des facteurs qui représentent certains aspects de la diversité ethnique ou linguistique des communautés de langue officielles dans une juridiction. Plus une juridiction est anglo-dominante (et par conséquent moins une juridiction est diversifiée d'une perspective linguistique et plus la communauté de langue officielle est dominante), plus le rapport offre/demande est faible. Et, simultanément, plus la communauté francophone de cette juridiction est diversifiée (et par conséquent plus sa cohérence linguistique et culturelle est faible), plus le rapport offre/demande est faible. Ce résultat démontre que des facteurs de cohérence linguistique et culturelle des deux communautés de langue officielle, l'une par rapport à l'autre, ont une association significative avec le rapport offre/demande, c'est-à-dire avec le taux de réalisation de services de santé en français dans une CFASM.

Ce résultat permet de mieux éclairer les observations de la première analyse. D'une part, la première analyse indique que le rapport offre/demande est plus grand lorsque la vitalité linguistique des CFASM est faible mais ce résultat n'est pas statistiquement significatif $(p=0,063)$. D'autre part, la deuxième analyse indique que des facteurs " environnementaux " autres que la vitalité langagière des CFASM ont une forte association statistique avec le rapport offre/demande sur une base juridictionnelle; ces facteurs représentent donc une source additionnelle de variance qui peut contribuer au fait que les résultats de la première analyse ne sont pas tout à fait statistiquement significatifs $(p=0,063)$. Il nous semble donc plausible de conclure que le rapport offre/demande est plus petit quand la vitalité linguistique des CFASM est faible, sous réserve que cette relation est sujette à une variance de cohérence 
linguistique et culturelle qui distingue les juridictions les unes des autres de façon singulière ${ }^{19}$.

\section{Interprétation}

Une étude précédente ${ }^{20}$ utilise les données probantes de l'enquête EVMLO de 2006 et du recensement canadien de 2006 pour élargir le schéma national du taux de couverture des soins en français du CCCFSM en situant des indices d'offre et de demande de soins de services de santé en français en fonction d'une mesure de masse critique de francophones, sur une base juridictionnelle (c'est-à-dire par province ou territoire) ${ }^{21}$. Il en ressort, relativement à la perception des francophones, un schème national cohérent où l'offre tombe en deçà de la demande et où l'offre et la demande sont plus grandes lorsque la mesure de masse critique de francophones est plus grande. Nous établissons ici que l'écart relatif observé entre l'offre et la demande a une forte association avec des facteurs qui reflètent la cohérence linguistique et culturelle des deux communautés de langues officielles l'une par rapport à l'autre. Ce résultat est consistant avec le fait que le schème national illustré dans la figure 1 s'inscrit dans un contexte de vitalité langagière; il est aussi cohérent avec :

(a) le travail de terrain de Forgues, Bahi et Michaud sur l'aménagement linguistique des services de santé dans les établissements de services de santé anglophones ou bilingues (hôpitaux) dans quatre provinces ${ }^{22}$, et

(b) des travaux théoriques ${ }^{23}$ basés sur les variables socio- et psycho-langagières du modèle du comportement psycho-

19 Par exemple le rapport offre/demande apparaît relativement petit pour le Manitoba et relativement grand pour le Terre-Neuve et Labrador (figure 1)

20 Louis Giguère, $o p$. cit.

21 Sylvie Lafrenière, op. cit.; Canada Canada, Statistique Canada, op. cit.; CCCFSM, "Pour un meilleur accès à des services de santé en français ", op. cit.

22 Eric Forgues, Boniface Bahi et Jacques Michaud, op. cit.; ces auteurs concluent que les niveaux de service reflètent l'état des rapports sociaux entre les francophones et les anglophones, et l'évolution de ces rapports.

Kenneth Deveau, Rodrigue Landry et Réal Allard, $o p$. cit.; Rodrigue Landry, Réal Allard et Kenneth Deveau, "A Macroscopic Intergroup Approach to 
langagier autonomisé et engagé de Landry et al. ${ }^{24}$ et de ses applications ${ }^{25}$.

Expliquant le modèle psycholangagier, Landry fait référence à une tension ou une relation dialectique entre un déterminisme social qui provient d'une collectivité de langue haute dont la vitalité est forte, et l'autodétermination d'une collectivité de langue basse dont la vitalité est faible ${ }^{26}$. Selon ce modèle, un endogroupe minoritaire est dans un rapport de force avec un exogroupe dominant. Selon lui, ce rapport de forces se vit dans un cadre idéologique, juridique et politique façonné au gré des forces de l'histoire et mettant en interaction des variables de nombre, de pouvoir et de statut, et ce contexte déterminerait le développement de la vitalité des communautés de langue officielle en situation minoritaire au Canada et les chances qu'une minorité linguistique puisse être et demeurer une entité distincte et active (ce qui, selon nous, comprendrait aussi l'accès à des services de santé dans la langue officielle d'une minorité).

Le schème national proposé ici (un schéma CCCFSM bonifié) est aussi cohérent avec un autre aspect du modèle ethnolinguistique d'autonomie culturelle de Deveau, Landry et Allard ${ }^{27}$. Ces auteurs affirment que, comme les CFASM sont issues historiquement d'une culture sociétale de langue française jadis partagée à l'époque du nationalisme canadien-français ${ }^{28}$ et qu'elles sont

the Study of Ethnolinguistic Development ", op. cit.; Rodrigue Landry, Réal Allard et Kenneth Deveau, "Un modèle macroscopique du développement psycholangagier en contexte intergroupe minoritaire ", op. cit.; Rodrigue Landry, op. cit.

24 Landry, Rodrigue et al., «Autodétermination du comportement langagier en contexte intergroupe minoritaire : un modèle conceptuel ", Francophonies d'Amérique, no 20, 2005, p. 63-78.

Kenneth Deveau, Rodrigue Landry et Réal Allard, op. cit.

Rodrigue Landry, op. cit.

Kenneth Deveau, Rodrigue Landry et Réal Allard, op. cit.

28 Selon Joseph Yvon Thériault, les " groupements nationalitaires » francophones subissent de fortes tendances à l'ethnicisation et ne seraient pas devenus des " groupes ethniques " suite à l'effondrement du Canada français ("Le difficile chemin de l'autonomie ", Minorités linguistiques et société, $\mathrm{n}^{\circ} 1,2012$, p. 37-50), mais ils persisteraient au travers d'une proposition de faire société en français au Canada et en Amérique du Nord (voir aussi Serge Bouchard, 
appuyées par un contexte politique et juridique fédéral commun, elles doivent partager plus d'éléments culturels communs que de différences même si elles ont évoluent sur des territoires géographiques et juridiques distincts. Cette perspective d'éléments culturels communs nous semble illustrée par le fait que les données sur l'offre et la demande de services de santé en français s'inscrivent sur des relations linéaires cohérentes lorsqu'elles sont prises en considération dans un contexte de vitalité linguistique. Il nous semble donc que le schème observé en figure 1 est une représentation concrète $\mathrm{du}$ modèle d'autonomie culturelle de Deveau, Landry et Allard $^{29}$ tel que perçu au travers du prisme des services de santé en français, et que ce schème valide la théorie linguistique sur laquelle ce modèle est fondé ${ }^{30}$. En contrepartie, les modèles d'autonomie culturelle de Deveau, Landry et Allard et de Landry nous semblent fournir des assises théoriques au schème national observé en figure 1 et valident une interprétation qui repose sur une dynamique de vitalité langagière.

Modélisations

Fort d'assises théoriques qui confirment la cohérence du schème national illustré en figure 1, nous proposons deux modélisations. La première est conceptuelle et elle consiste à établir une concordance entre les trois acteurs et les trois composantes du modèle ethno-langagier de Landry ${ }^{31}$ et les trois phases du schéma $\mathrm{CCCFSM}^{32}$. La deuxième consiste à explorer le schème national de la figure 1 d'une perspective systémique et à le modéliser en diagramme de phase où les relations linéaires d'offre et de demande de services en français de la figure 1 représentent des trajectoires structurantes/contraignantes de l'offre et de la demande de services en français.

"Les francophones d'Amérique : une communauté de destins ", Québec, Forum de la francophonie canadienne, mai 2012).

30 Rodrigue Landry, op. cit., p. 163.

31 Ibid.

32 CCCFSM, "Pour un meilleur accès à des services de santé en français ", op. cit. 
Premier exercice de modélisation : concordance des acteurs et des composantes du modèle ethno-langagier de Landry avec les phases du schéma national de services de santé en français du CCCFSM

La première composante du modèle de Landry, et la plus fondamentale, est la proximité socialisante qui est basée sur les individus et les familles comme premiers agents d'une socialisation langagière primaire et qui assure la transmission intergénérationnelle de la langue. Cette composante est limitée aux fonctions sociales de l'endogroupe dans la sphère privée et elle ne trouve aucun prolongement dans la sphère publique à moins de pouvoir être appuyée par les deux autres composantes de l'autonomie culturelle (discutées ci-dessous). Cette situation se retrouve dans une CFASM où la vitalité langagière est basse et correspondrait à la phase de sensibilisation du CCCFSM où les CFASM doivent surtout concentrer leurs actions sur une prise de conscience de la problématique des services de santé en français.

La deuxième composante du modèle de Landry est la complétude institutionnelle qui est basée sur les leaders communautaires, les gestionnaires d'institutions, les membres d'organismes communautaires et les membres de la structure de gouvernance qui représentent la société civile, l'intermédiaire entre la communauté non organisée socialement et l'État. Par la voie de ces animateurs, la société civile peut agir politiquement et orienter les actions du groupe. Cette situation qui se retrouverait dans une CFASM où la vitalité langagière est plus forte pourrait correspondre à la phase de structuration du CCCFSM où l'action doit aussi comprendre une prise de conscience de la problématique des services de santé en français mais surtout se concentrer sur le développement soutenu des services offerts en français et sur la structuration et l'institutionnalisation de l'offre de ces services.

La troisième composante du modèle de Landry est la légitimité idéologique qui représente l'appui de l'État et de ses citoyens à l'autonomie culturelle de la minorité et qui se concrétise par la consécration de droits et la création de programmes et de services. Cette reconnaissance, à son tour, influe sur les représentations sociales des membres de la minorité. Cette situation qui se 
retrouve dans une CFASM où la vitalité langagière est forte pourrait correspondre à la phase de structuration où le CCCFSM suggère que les francophones, les professionnels de la santé, les institutions existantes et les autorités gouvernementales se concentrent sur la protection des acquis et l'adaptation aux nouvelles technologies et façons de faire.

Comme la proximité socialisante nous semble représenter une composante qui s'applique à tous les CFASM du Canada et qu'elle a un fondement d'action commun, nous lui avons donné un poids important pour chaque phase CCCFSM dans le tableau de correspondance donné ci-dessous (tableau 5).

\section{Tableau 5}

Modélisation des composantes du modèle ethno-langagier de Landry en fonction des phases évolutives du schéma national de services en santé en français du CCCFSM

\begin{tabular}{|c|c|c|c|}
\hline & \multicolumn{3}{|c|}{ la vitalité langagière augmente $\longrightarrow$ CFASM en phase } \\
\hline $\begin{array}{l}\text { Acteurs et composantes } \\
\text { du modèle de Landry }\end{array}$ & $\begin{array}{c}\ldots \text { de } \\
\text { sensibilisation }\end{array}$ & ... de structuration & ... de consolidation \\
\hline $\begin{array}{l}\text { Les individus et les } \\
\text { familles agissant sur la } \\
\text { proximité socialisante }\end{array}$ & $\begin{array}{c}\text { Agir constamment } \\
\text { sur cette } \\
\text { composante }\end{array}$ & $\begin{array}{c}\text { Agir fréquemment } \\
\text { sur cette } \\
\text { composante }\end{array}$ & $\begin{array}{l}\text { Agir fréquemment } \\
\text { sur cette } \\
\text { composante }\end{array}$ \\
\hline $\begin{array}{l}\text { Les leaders de la } \\
\text { société civile agissant } \\
\text { sur la complétude } \\
\text { institutionnelle }\end{array}$ & $\begin{array}{c}\text { Agir } \\
\text { occasionnellement } \\
\text { sur cette } \\
\text { composante }\end{array}$ & $\begin{array}{l}\text { Agir fréquemment } \\
\text { sur cette } \\
\text { composante }\end{array}$ & $\begin{array}{l}\text { Agir fréquemment } \\
\text { sur cette } \\
\text { composante }\end{array}$ \\
\hline $\begin{array}{l}\text { L'État et ses citoyens } \\
\text { agissant sur la légitimité } \\
\text { idéologique }\end{array}$ & $\begin{array}{l}\text { Agir rarement sur } \\
\text { cette composante }\end{array}$ & $\begin{array}{c}\text { Agir } \\
\text { occasionnellement } \\
\text { sur cette } \\
\text { composante }\end{array}$ & $\begin{array}{l}\text { Agir fréquemment } \\
\text { sur cette } \\
\text { composante }\end{array}$ \\
\hline
\end{tabular}

Les CFASM en phase de sensibilisation agiraient surtout sur la composante de proximité socialisante. Les CFASM en phase de structuration et de consolidation agiraient elles aussi sur la composante de complétude institutionnelle, alors que la légitimité idéologique aurait sa plus forte expression dans la CFSASM de consolidation du Nouveau-Brunswick. 
Cette représentation bonifie une des conclusions de notre article précédent qui suggère que les CFASM inventorient leurs bonnes pratiques, leurs approches d'évaluation et de partage de connaissances en tenant compte de leur contexte de sensibilisation, de structuration ou de consolidation. Selon nous, non seulement les CFASM doivent-elles choisir de meilleures pratiques qui tiennent compte de ces contextes, mais aussi elles doivent choisir des façons de faire ou des approches d'intervention qui reflètent l'importance relative des trois composantes du modèle de Landry pour cette CFASM. Au Nouveau-Brunswick par exemple, l'approche devra agir sur les trois composantes du modèle de Landry et devra être plus globale; elle devra reposer sur un plan stratégique de plus longue haleine qui s'investira dans une programmation santé intégratrice qui troque avec le système d'égal à égal; la CFASM pourra y proposer des interventions au système de santé. Par ailleurs, dans une CFASM de sensibilisation comme la Colombie-Britannique l'approche reposera surtout sur une composante de proximité socialisante et son plan stratégique sera à plus court terme et visera à s'investir dans des projets d'engagement communautaire tout en gardant l'œil sur les occasions qui émanent " spontanément » du système de santé (la CFASM devra se positionner pour saisir les occasions émergentes, tout en sensibilisant; en fait, une approche considérablement plus ad hoc).

Deuxième exercice de modélisation : explorer le schème national en diagramme de phase (une perspective systémique)

Si nous considérons le schème national de la figure 1 selon des trajectoires contraignantes (ou structurantes) de l'offre et de la demande de services en français dans les CFASM, une modélisation en "diagramme de phase " est possible. Provenant du secteur des sciences chimiques et physiques, cette approche systémique a obtenu une légitimité en sciences écologiques ${ }^{33}$,

33 Vladimir B. Verbitskii et Vladimir G. Tereshchenko, "Structural Phase Diagrams of Animal Communities in Assessment of Freshwater Ecosystem Conditions ", Hydrobiologia, n ${ }^{\circ} 322$, 1996, p. 277-382. 
économiques ${ }^{34}$, géographiques ${ }^{35}$ et sociales $^{36}$ quand on veut explorer l'évolution spatiale et temporelle de phénomènes complexes $^{37}$.

Selon cette modélisation, l'offre et la demande de services de santé en français augmenteraient ou diminueraient le long des deux trajectoires entrevues par le biais de la masse critique de francophones ${ }^{38}$. Ceci ne revient pas à dire que la masse critique (un indicateur de vitalité langagière) fait augmenter l'offre et la demande; ceci signifie plutôt que le système considéré se comporte de façon telle que les relations et les interactions de l'ensemble des variables socio-démographiques du système donnent cours à une configuration singulière. S'il arrivait que les efforts de développement communautaire d'une CFASM portent fruit et que celle-ci s'épanouisse, il y aura glissement de la masse critique de francophones le long de l'axe des « $\mathrm{X}$ » et augmentation de concomitance de l'offre et de la demande de services en français le long des deux trajectoires comme l'illustre la figure 1 . Selon cette perspective systémique, une CFASM n'est donc pas

34 Ronald Shone, Economic Dynamics : Phase Diagrams and their Economic Application, Cambridge (NY), Cambridge University Press, 2002; http:// www.econ.uniurb.it/materiale/5773_Shone\%20-\%20Economic\%20

Dynamics.pdf, site consulté le 3 juin 2013.

35 Yves Guermond, The Modeling Process in Geography: From Determinism to Complexity, Hoboken, John Wiley and Sons, 2008.

36 Teimuraz Matcharashvili, Tamaz Chelidze et Manana Janiashvili, "Identification of Complex Processes Based on Analysis of Phase Space Structures ", dans Jim Byrnes (dir.), NATO Security through Science Series : Imaging for Detection and Identification, Dordrecht (Pays-Bas), Springer, 2007, p. 207-242.

37 Teimuraz Matcharashvili, Tamaz Chelidze et Manana Janiashvili, ibid. Le CCCFSM lui-même avait proposé que les services en français augmentent ou diminuent le long d'une trajectoire commune à partir d'une position qui est propre à chaque province, territoire, région de santé ou communauté selon les conditions géographiques, démographiques, sociales et historiques qui y existent et qui s'y développent (CCCFSM, " Pour un meilleur accès à des services de santé en français ", op. cit.)

38 Sous réserve que cette relation est sujette à des variations de cohérence linguistique et culturelle qui distinguent les juridictions les unes des autres de façon singulière; cet exercice de modélisation doit donc être interprété dans le contexte de l'ensemble des CFASM pour dégager des principes de base, et non pas par référence à une CFASM particulière. 
" libre » de se déplacer dans n'importe quelle direction dans l'espace défini par la figure 1; elle est contrainte par un environnement où le développement des services de santé en français doit suivre une trajectoire prescrite. Même si, par exemple, la CFASM de la Colombie-Britannique investit dans un projet de services de santé pour faire augmenter ou pour " forcer " l'offre à la verticale dans le schème illustré en figure 1 en appliquant des efforts d'organisation de services prononcés, cela n'aura qu'un impact limité ou temporaire, car les contraintes du cadre national structurant interviendraient pour rétablir l'équilibre offre / demande prescrit par l'état du système dans son ensemble. Une initiative d'organisation de services sophistiquée ${ }^{39}$ échouerait donc à moins qu'elle soit appropriée au contexte de la demande pour cette CFASM. Cette perspective semble être confirmée par les faits en Colombie-Britannique. Le bilan des initiatives réalisées lors des huit premières années d'activité de RésoSanté Colombie-Britannique mis à jour lors de la planification stratégique de 2011 ainsi que le bilan des initiatives réalisées à la suite de l'Appel d'offres 2010-2013 indiquent que sept initiatives santé sur neuf qui sont associées aux régies de santé et qui représentent surtout des initiatives d'organisation de services ont cessé d'exister suite à l'expiration du projet ${ }^{40}$. Par contraste, neuf initiatives santé sur dix associées aux partenaires communautaires et qui représentent surtout des initiatives de sensibilisation et de promotion de la santé ont duré ${ }^{41}$. La leçon nous semble donc que, pour maximiser le retour sur son investissement, le réseau de santé de la Colombie-Britannique qui opère dans une CFASM

39 Qui pourrait être considérée ailleurs comme une " meilleure pratique ".

40 Projets autisme, diabète, ateliers de toxicomanie, clinique pour les démunis francophones, trousse pour les nouvelles familles, site Web et accès aux services, services aux immigrants; les initiatives durables sont deux sites Web sur la santé mentale.

41 Projets Grandir C.-B., Mes Choix Ma Santé, trousse Bébés aux couches, ateliers d'adaptation culturelle, atelier "Soignez vos patients en français ", accompagnement des clients du Centre Communautaire La Boussole, équipe multidisciplinaire pour les clients du Centre Communautaire La Boussole, Cour atout, ateliers de nutrition en milieux scolaire et pré-scolaire; l'initiative non durable est la conférence et le site Web "Parlons santé mentale ». 
en phase de sensibilisation doit aligner ses interventions en fonction du contexte de l'offre et de la demande qui prévaut dans sa communauté, favorisant une prépondérance de projets de sensibilisation. Quand le réseau de santé de la ColombieBritannique s'investit dans des modèles d'organisation de services médicaux, de thématiques santé ou de meilleures pratiques provenant de courants dominants avec le système de santé, le retour sur son investissement est limité; quand il s'investit dans des initiatives communautaires de santé, le retour sur l'investissement est considérable pour deux raisons : (a) parce que le projet dure, et (b) parce qu'il a pour effet une sensibilisation de la communauté à l'importance de services de santé linguistiquement et culturellement appropriés (ce qui ultimement favorisera l'épanouissement de la CFASM, et fera augmenter la demande et l'offre en concomitance). La même leçon nous semble aussi valide pour le programme du Bureau d'appui aux communautés de langue officielle (BACLO) de Santé Canada qui, à un niveau national, doit appuyer les réseaux de santé qui opèrent dans des milieux divers où les contextes de l'offre et de la demande sont radicalement différents. Le programme du BACLO s'inscrit dans le contexte d'une Feuille de route pour les langues officielles qui met l'accent sur l'épanouissement des CFASM et qui est guidé par un Plan fédéral d'action sur les langues officielles ${ }^{42}$ reposant sur quatre principes directeurs pour rendre plus efficace la stratégie préconisée $^{43}$ :

- le besoin d'agir autant sur l'offre que sur la demande

- la nécessité d'un effort concerté

- la reconnaissance des différences régionales

- l'importance de la participation des communautés

Le BACLO ne préconise donc pas une approche de santé primaire médicale; il suggère plutôt une approche globale, concertée et flexible où la sensibilisation et la proximité socialisante jouent un rôle important. Cependant cette approche est

\footnotetext{
$\overline{42}$ CCCFSM, « Rapport au ministre fédéral de la santé. Pour un nouveau leadership en matière d'amélioration des services de santé en français ", Ottawa, 2007.

43 Et de ce fait aussi réaliser un bon retour sur leur investissement.
} 
mal reflétée dans le Modèle Logique 2014-2018 qu'il propose ${ }^{44}$ puisque les deux résultats immédiats, les trois produits bénéficiaires et les cinq activités bénéficiaires reflètent tous une approche de services «santé primaire ». Une révision pourrait favoriser un meilleur retour sur son investissement.

Reconnaissant qu'une approche en diagramme de phase met beaucoup l'accent sur les contraintes d'un système, cette approche n'exclut pas l'action sur ces contraintes mais elle nous force à reconnaître qu'une telle action est considérablement plus globale et à plus long terme. Il serait donc possible de modifier les contraintes du cadre national structurant illustré en figure 1 et de réduire l'écart qui existe entre l'offre et la demande (autrement dit de mieux satisfaire aux attentes des francophones des CFASM) en effectuant un déplacement de l'ensemble de la trajectoire de l'offre vers le haut. Comme nous avons observé que le rapport offre /demande est fortement lié à des facteurs associés avec la cohérence linguistique et culturelle des deux communautés de langue officielle, la modification du cadre national structurant (l'environnement global qui détermine la position des trajectoires d'offre et de demande) semble donc possible au travers d'une action concertée ciblant les facteurs qui régissent ce rapport de forces. C'est donc une entreprise à long terme qui sous-tend une action nationale dans le contexte de ce que Landry appelle un rapport de forces entre un déterminisme social qui provient de la collectivité de langue haute et une autodétermination qui provient de la collectivité de langue basse ${ }^{45}$. Cette interprétation du modèle en diagramme de phase présenté ici suggère que les interventions des réseaux de santé des CFASM et de leurs partenaires peuvent se baser principalement sur deux volets stratégiques distincts mais complémentaires :

- l'action régionale sur la dynamique du cadre structurant, une action à court ou à moyen terme qui se joue à un niveau plus proximal dans un contexte régional et qui

$\overline{44}$ Canada, Santé Canada, " Programme de contribution pour les langues officielles en santé - Présentation aux communautés francophones en situation minoritaire ", Ottawa, 15 juillet 2013. 
repose sur des partenaires qui collaborent pour favoriser la croissance de la vitalité linguistique et le développement de ces communautés en tant que communautés francophones $^{46}$; et

- l'action nationale sur l'évolution du cadre structurant, une action à long terme pour faire déplacer les trajectoires de la figure 1 vers le haut. Ce travail doit se faire de concert avec d'autres CFASM et avec d'autres organismes ou institutions de niveau national, y compris des partenaires dans d'autres secteurs ou d'autres régions (par exemple la communauté francophone majoritaire du Québec), et y compris des partenaires de la communauté de langue officielle majoritaire anglophone.

L'action sur deux volets stratégiques représente un défi pour le développement des CFASM et des réseaux de santé qui s'y trouvent : car comment allouer ses efforts entre le réseautage régional pour agir à court terme sur le volet de la dynamique de développement communautaire et le réseautage organisationnel national pour agir à long terme sur l'évolution des contraintes d'un cadre national structurant dominé par la collectivité anglophone?

\section{L'action sur les contraintes du cadre national structurant et le rapport de forces des communautés de langues officielles}

Létude de terrain de Forgues, Bahi et Michaud sur les facteurs sociaux qui influencent l'offre de services en français dans quatre hôpitaux desservant des CFASM peut nous éclairer en ce qui a trait aux contraintes qui préviennent la prestation des services de santé en français dans les CFASM. Les contraintes qu'ils ont identifiées sont les suivantes : 1- les attitudes des anglophones,

$\overline{46}$ Pour la Colombie-Britannique par exemple, une CFASM en phase de sensibilisation, cette action doit surtout cibler des interventions qui sont compatibles avec la croissance de la vitalité linguistique et le développement de ces communautés en tant que communautés francophones, c'est-à-dire selon la composante de proximité socialisante du modèle de Landry. L'action sur des composantes de complétude institutionnelle et de légitimité idéologique donnerait peu de résultats. 
2- les attitudes des professionnels francophones et francophiles, et 3- les attitudes des francophones des CFASM. Du côté des anglophones, on n'est pas enclin à reconnaitre le droit des francophones à recevoir des services en français, surtout pour les francophones qui sont bilingues, pour les raisons suivantes :

Les commentaires de plusieurs répondants anglophones vont plutôt dans le sens d'un questionnement de ce droit. On comprend mal comment, dans un contexte multiculturel, on fait plus d'efforts pour les francophones que pour les autres minorités ethniques. On met ainsi sur le même pied d'égalité le droit des immigrants et celui des francophones, oubliant l'existence de la Loi sur les langues officielles. Le droit des patients de recevoir des services en français se définit dans une perspective de gestion : la langue de communication ayant une influence certaine sur la qualité des soins offerts, il n'y a pas de différence entre un patient francophone et un nouvel arrivant qui parle une autre langue que l'anglais ${ }^{47}$.

On voit donc que l'attitude des anglophones en situation majoritaire joue un rôle déterminant en ce qui a trait aux niveaux de services de santé en français. Comme les attitudes sociales et politiques qui prévalent à l'heure actuelle au Canada en milieu anglophone (celles de l'exogroupe dominant) semblent se diriger inexorablement vers le multiculturalisme au fur et à mesure que le Canada devient, de plus en plus, un pays d'immigrants ${ }^{48}$, un déterminisme social multiculturel, basé sur le nombre, exerce une pression vers le bas sur la trajectoire de l'offre de services de la figure 1 (une trajectoire qui représente ce que la communauté de langue officielle dominante est prête à offrir à une CFASM). Les dossiers canadiens nationaux de la dualité linguistique et des langues officielles ${ }^{49}$ ainsi les attitudes sociales et politiques qui prévalent à l'heure actuelle chez les francophones (celles de l'endogroupe minoritaire, y compris la demande exprimée pour

\footnotetext{
$47 \quad$ Éric Forgues, Boniface Bahi et Jacques Michaud, op. cit., p. 13.

48 Et que ces immigrants adoptent principalement la langue de la majorité.

49 Ces dossiers sont principalement fédéraux et leur impact est mitigé selon les conditions du partage des responsabilités et des pouvoirs entre les acteurs fédéraux, provinciaux et territoriaux; comme le secteur de la santé est considéré comme étant une juridiction provinciale et territoriale, la contrepression est faible.
} 
des services en français), exercent une contre-pression vers le haut. L'action sur les contraintes du cadre structurant doit donc cibler les attitudes du partenaire dominant dans l'esprit de rapport de forces, tout en profitant des avantages de poids démographiques qui existent pour les francophones au niveau national. Les conditions de réussite d'une telle entreprise pour les CFASM et leurs partenaires pourraient comprendre un ou plusieurs des éléments suivants :

1- Rechercher l'appui du Québec en ce qui a trait aux services de santé en français dans les CFASM; de par son poids démographique, l'appui du Québec, des professionnels de la santé québécois et des Québécois francophones, est essentiel pour agir sur les facteurs qui affectent le cadre national structurant.

2- Rechercher l'appui des grands organismes de santé qui ont à cour les valeurs canadiennes exprimées par les lois sur les langues officielles ainsi que le développement de la francophonie. Ceci comprend les associations et les ordres professionnels nationaux et les organisations nationales de santé où les francophones devraient avoir une représentation importante. Ils peuvent y exercer un leadership.

3- Encourager les professionnels de la santé francophones, et en particulier ceux du Québec et du Nouveau-Brunswick, à devenir membres des associations et des ordres professionnels nationaux et à s'y engager. Les professionnels francophones sont souvent sous-représentés dans les organismes nationaux et ceux-ci sont souvent anglo-dominés (les francophones se joignent souvent à des associations francophones "parallèles »). Il est essentiel que les professionnels francophones prennent la place qui leur revient au sein de leurs organismes nationaux et qu'ils y appuient le développement des services en français dans les CFASM.

4- Choisir un positionnement pour les CFASM qui les distingue bien des autres minorités nationales (les communautés immigrantes et autochtones). L'environnement dans lequel les CFASM opèrent est déterminé par un exogroupe 
qui, dans certaines juridictions dites unilingues anglophones, entrevoit souvent les minorités linguistiques à travers une lentille multiculturelle. Un positionnement distinctif est suggéré par le travail de Thériault, qui décrit les CFASM comme des groupes intermédiaires, "nationalitaires ", situés entre l'ethnie et la nation ${ }^{50}$, par le travail de Poirier qui positionne les CFASM entre le pôle immigrant et le pôle "Première Nation " d'un continuum ethnie-nation ${ }^{51}$, et par le travail de Cardinal et González Hidalgo qui précisent des droits distincts pour les trois minorités linguistiques et culturelles de la société canadienne; ces droits sont le droit à la non-discrimination (ou l'inclusion) pour les ethnies, le droit à la complétude institutionnelle pour les CFASM, et le droit à l'autonomie pour les minorités nationales autochtones ${ }^{52}$. Les CFASM et leurs réseaux santé doivent donc sensibiliser les communautés francophones, anglophones, allophones et immi-

Joseph Yvon Thériault, "De la nation à l'ethnie. Ssociologie, société et communautés francophones minoritaires ", Sociologie et sociétés, vol. 26, $\mathrm{n}^{\circ} 1$, 1994, p. 15-32.

Johanne Poirier, «Au-delà des droits linguistiques et du fédéralisme classique : favoriser l'autonomie institutionnelle des francophones minoritaires du Canada ", dans Joseph Yvon Thériault, Anne Gilbert et Linda Cardinal (dir.), L'espace francophone en milieu minoritaire au Canada: nouveaux enjeux, nouvelles mobilisations, Montréal, Fides, 2009, p. 513-562; Johanne Poirier, "Autonomie politique et minorités francophones du Canada : réflexions sur un angle mort de la typologie classique de Will Kymlicka ", Minorités linguistiques et société, $\mathrm{n}^{\circ} 1,2012$, p. 66-89.

52 Linda Cardinal et Eloísa González Hidalgo, "L'Autonomie des minorités francophones hors Québec au regard du débat sur les minorités nationales et les minorités ethniques ", Minorités linguistiques et société, $\mathrm{n}^{\circ}$ 1, 2012, p. 51-65. Will Kymlicka approche le problème d'une perspective différente mais conclut aussi que " the success of the Canadian model of countermajoritarian democracy depends on keeping these three pillars distinct [...] Aboriginal issues addressed through the lens of Section 25 and 35 of the constitution [...] The rights of the OLMCs are addressed through the lens of Sections 16-23 of the Constitution and the associated Official Languages Act [...] The claims of immigrant-origin ethnic groups are addressed through the lens of Section 27 of the Constitution and the associated Multiculturalism Act " ("A New Deal for LMC's? Three Challenges ", Minorités linguistiques et société, $\mathrm{n}^{\circ} 1,2012$, p. 252). 
grantes et se positionner dans un contexte des trois piliers minoritaires de l'identité canadienne. Ce positionnement nous semble essentiel pour contrecarrer la tendance qu'a la communauté anglophone à interpréter le dossier francophone à l'intérieur du dossier multiculturel. Le défi consiste non seulement à réaliser ce positionnement au niveau national mais encore à le faire transparaître aux niveaux provinciaux et territoriaux quel que soit le partage des responsabilités et des pouvoirs entre les acteurs fédéraux, provinciaux et territoriaux.

Ce positionnement pourrait comprendre un ou plusieurs des éléments suivants :

- mettre à jour les documents de vision, mission, etc. des organismes francophones des CFASM pour refléter un positionnement singulier dans le contexte des minorités canadiennes;

- sensibiliser le leadership des systèmes de santé et l'encourager à adopter une politique claire à l'égard des services de santé pour les trois minorités, y compris sa minorité de langue officielle.

On notera que les CFASM de sensibilisation sont la clé pour bien effectuer ce positionnement. C'est chez les CFASM de sensibilisation que le déséquilibre du rapport de forces entre les deux communautés de langue officielle est le plus grand. D'une certaine façon, ces CFASM représentent le futur des autres CFASM si la tendance démographique canadienne actuelle maintient sa démarche vers le développement d'une société de plus en plus diversifiée et multiculturelle. Il faut donc consacrer plus de travail de recherche aux les CFASM de sensibilisation pour mieux comprendre cet enjeu et pour aider à développer un positionnement national qui pourra être proposé à l'exogroupe dominant et assumé par lui, quelle que soit la juridiction. Le même raisonnement s'applique dans le cas de "meilleures pratiques ". Une meilleure pratique développée dans une CFASM de sensibilisation et qui tient bien compte du contexte multiculturel peut être utile pour l'ensemble des CFASM. Le corollaire 
n'est pas nécessairement vrai. Par exemple, la Société Santé en français et certains réseaux de structuration ou de consolidation préconisent souvent l'approche dite de l'offre active de services en français. Une approche d'offre active réussit bien lorsqu'il y a un alignement des dossiers de dualité linguistique et des dossiers multiculturels, c'est-à-dire lorsque le français est la deuxième ou possiblement la troisième langue parlée dans une juridiction. Pour l'Alberta, la Colombie-Britannique, le Manitoba, le Nunavut, la Saskatchewan et le Terre-Neuve et Labrador cependant (voir le tableau 6 ci-dessous), il est plus difficile de justifier une offre active de services de santé en français auprès d'un système de santé d'une façon globale.

Tableau 6

Rang du français dans la juridiction en tant que langue parlée le plus souvent à la maison (Recensement de 2006)

\begin{tabular}{|l|c|c|c|c|}
\hline Rang & 2 & 3 & 5 & 9 \\
\hline Juridiction & $\begin{array}{c}\text { IPE, NB, NE, } \\
\mathrm{ON}, \mathrm{YU}\end{array}$ & $\mathrm{MA}, \mathrm{NU}, \mathrm{TNL}$ & $\mathrm{AB}, \mathrm{SA}$ & $\mathrm{CB}$ \\
\hline
\end{tabular}




\section{Bibliographie}

Bouchard, Serge, «Les francophones d'Amérique : une communauté de destins ", Québec, Forum de la francophonie canadienne, mai 2012.

Canada, Santé Canada, "Programme de contribution pour les langues officielles en santé - Présentation aux communautés francophones en situation minoritaire », Ottawa, 15 juillet 2013.

Canada, Statistique Canada, "Portrait des communautés de langue officielle au Canada : recensement de 2006 ", Ottawa, 2009, DVD.

Cardinal, Linda et Eloísa González Hidalgo, "L'autonomie des minorités francophones hors Québec au regard du débat sur les minorités nationales et les minorités ethniques ", Minorités linguistiques et société, $\mathrm{n}^{\circ} 1,2012$, p. 51-65.

CCCFSM [Comité consultatif des communautés francophones en situation minoritaire], "Pour un meilleur accès à des services de santé en français ", Ottawa, Fédération des communautés francophones et acadiennes du Canada, 2001.

CCCFSM [Comité consultatif des communautés francophones en situation minoritaire], «Rapport au ministre fédéral de la santé. Pour un nouveau leadership en matière d'amélioration des services de santé en français ", Ottawa, 2007.

CNFS et SSF [Consortium national de formation en santé et Société Santé en français], "Introduction et mise en contexte ", dans Le Secrétariat national du Consortium national de formation en santé Société, langue et santé : les barrières à l'accès aux services de santé pour les communautés francophones minoritaires, $2^{\mathrm{e}}$ Forum national de recherche sur la santé des communautés francophones en situation minoritaire, Ottawa, 2008, p. 15-18.

Deveau, Kenneth, Rodrigue Landry et Réal Allard, «Utilisation des services gouvernementaux de langue française. Une étude auprès des Acadiens et francophones de la Nouvelle-Écosse sur les facteurs associés à l'utilisation des services gouvernementaux en français ", Ottawa, Institut canadien de recherche sur les minorités linguistiques, 2009.

Forgues, Éric, Boniface Bahi et Jacques Michaud, «L'offre de services en français en contexte francophone minoritaire ", Ottawa, Institut canadien de recherche sur les minorités linguistiques, 2011.

Giguère, Louis, «Les services de santé en français pour les communautés francophones et acadiennes en situation minoritaire du Canada : bonification du schéma du CCCFSM (2001) ", Nouvelles perspectives en sciences sociales, vol. 9, $\mathrm{n}^{\circ}$ 1, 2013, p. 319-345. 
Guermond, Yves, The Modeling Process in Geography: From Determinism to Complexity, Hoboken, John Wiley and Sons, 2008.

Houle, René et Jean-Pierre Corbeil, « Portrait statistique de la population immigrante de langue française à l'extérieur du Québec (1991 à 2006) », Ottawa, Statistique Canada, 2010.

Kymlicka, Will, "A New Deal for LMC's? Three Challenges », Minorités linguistiques et société, $\mathrm{n}^{\circ} 1,2012$, p. 248-258.

Lafrenière, Sylvie "L'accès aux services de soins de santé : le potentiel analytique de l'Enquête sur la vitalité des minorités de langue officielle ", dans Le Secrétariat national du Consortium national de formation en santé Société, langue et santé : les barrières à l'accès aux services de santé pour les communautés francophones minoritaires,

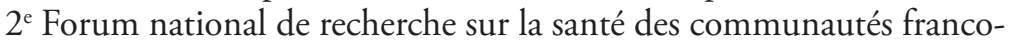
phones en situation minoritaire, Ottawa, 2008, p. 31-32.

Landry, Rodrigue, «Autonomie culturelle, cultures sociétales et vitalité des communautés de langue officielle en situation minoritaire au Canada ", Minorités linguistiques et société, $\mathrm{n}^{\circ}$ 1, 2012, p. 159-179.

Landry, Rodrigue, Réal Allard et Kenneth Deveau, "A Macroscopic Intergroup Approach to the Study of Ethnolinguistic Development ", International Language of the Sociology of Language, vol. 185, 2007, p. 225-253.

Landry, Rodrigue, Réal Allard et Kenneth Deveau, «Un Modèle macroscopique du développement psycholangagier en contexte intergroupe minoritaire ", Diversité urbaine, hors série, automne 2008, p. 45-68.

Landry, Rodrigue et al., "Autodétermination du comportement langagier en contexte intergroupe minoritaire : un modèle conceptuel ", Francophonies d'Amérique, no 20, 2005, p. 63-78.

Matcharashvili, Teimuraz, Tamaz Chelidze et Manana Janiashvili, "Identification of Complex Processes Based on Analysis of Phase Space Structures ", dans Jim Byrnes (dir.), NATO Security through Science Series: Imaging for Detection and Identification, Dordrecht (Pays-Bas), Springer, 2007, p. 207-242.

Poirier, Johanne, "Au-delà des droits linguistiques et du fédéralisme classique : favoriser l'autonomie institutionnelle des francophones minoritaires du Canada ", dans Joseph Yvon Thériault, Anne Gilbert et Linda Cardinal (dir.), L'espace francophone en milieu minoritaire au Canada: nouveaux enjeux, nouvelles mobilisations, Montréal, Fides, 2009, p. 513-562.

Poirier, Johanne, "Autonomie politique et minorités francophones du Canada : réflexions sur un angle mort de la typologie classique de Will Kymlicka ", Minorités linguistiques et société, n 1, 2012, p. 66-89. 
Shone, Ronald, Economic Dynamics: Phase Diagrams and their Economic Application, Cambridge (NY), Cambridge University Press, 2002; http://www.econ.uniurb.it/materiale/5773_Shone $\% 20-\% 20$ Economic\%20Dynamics.pdf, site consulté le 3 juin 2013.

Simpson, Edward H., "Measurement of diversity ", Nature, n 163, 1949, p. 688.

Thériault, Joseph Yvon, "De la nation à l'ethnie : sociologie, société et communautés francophones minoritaires ", Sociologie et sociétés, vol. 26, no 1, 1994, p. 15-34.

Thériault, Joseph Yvon, "Le difficile chemin de l'autonomie ", Minorités linguistiques et société, $\mathrm{n}^{\circ} 1,2012$, p. 37-50.

Verbitskii, Vladimir B. et Vladimir G. Tereshchenko, «Structural Phase Diagrams of Animal Communities in Assessment of Freshwater Ecosystem Conditions ", Hydrobiologia, no 322, 1996, p. 277-382.

Zar, Jerrold H., Biostatistical Analysis, $2^{\mathrm{e}}$ édition, Upper Saddle River (NJ), Prentice Hall, 1984. 